\title{
Scientific Workshop Proceedings of the XP2015
}

$16^{\text {th }}$ International Conference on Agile Software Development

25-29 May, 2015, Helsinki, Finland

General Scientific Workshop Chairs: Casper Lassenius and Daniela S. Cruzes

Scientific Workshop Proceedings Chair: Eero Laukkanen

Conference Chair: Maria Paasivaara

\section{3rd International Workshop on \\ Large-Scale Agile Development}

Workshop Organizers: Torgeir Dingsøyr and Nils Brede Moe

\section{1st International Workshop on \\ Requirements Engineering in Agile Development (READ) \\ Workshop Organizers: Sabrina Marczak, Maya Daneva, Ville T. Heikkilä, \\ Nils Brede Moe and Knut H. Rolland}

\section{International Workshop on \\ Refactoring \& Testing (RefTest 2015)}

Workshop Organizers: Steve Counsell, Francesca Arcelli Fontana,

Alessandro Murgia and Roberto Tonelli

ACM COPYRIGHT NOTICE. Copyright (C 2015 by the Association for Computing Machinery, Inc. Permission to make digital or hard copies of all or part of this work for personal or classroom use is granted without fee provided that copies are not made or distributed for profit or commercial advantage and that copies bear this notice and the full citation on the first page.

To copy otherwise, to republish, to post on servers or to redistribute to lists, requires prior specific permission and/or a fee. 


\section{Table of Contents}

\section{3rd International Workshop on Large-Scale Agile Development}

\section{Agile Portfolio Management at Finnish Broadcasting Company Yle}

Maarit Laanti, Nitor Delta

Rami Sirkia, Nitor Delta

Mirette Kangas, Finnish Broadcasting Company

\section{Lean Startup: Why Large Software Companies Should Care}

Henry Edison, Free University of Bozen-Bolzano

Xiaofeng Wang, Free University of Bozen-Bolzano

Pekka Abrahamsson, Norwegian University of Science Technology

\section{Towards Continuous Validation of Customer Value}

Helena Holmstrom Olsson, Malmo University

Jan Bosch, Chalmers University of Technology

\section{1st International Workshop on Requirements Engineering in Agile Development (READ) \\ Re-conceptualizing requirements engineering: \\ Findings from a large-scale, agile project \\ Tor Erlend Fægri, SINTEF ICT \\ Nils Brede Moe, SINTEF ICT \\ 'Desperately' Seeking Research on Agile Requirements in the Context of Large-Scale Agile Projects \\ Knut H. Rolland, Westerdals Oslo School of Arts, Communication and Technology \& SINTEF ICT}

A Reflection on Agile Requirements Engineering:

Solutions Brought and Challenges Posed

Irum Inayat, University of Malaya

Lauriane Moraes, PUCRS University

Maya Daneva, University of Twente

Siti Salwah Salim, University of Malaya 


\section{International Workshop on Refactoring \& Testing (RefTest 2015)}

On Experimenting Refactoring Tools to Remove Code Smells

Francesca Arcelli Fontana, University of Milano-Bicocca Marco Mangiacavalli, University of Milano-Bicocca

Domenico Pochiero, University of Milano-Bicocca Marco Zanoni, University of Milano-Bicocca

Mutation Testing as a Safety Net for Test Code Refactoring

Ali Parsai, University of Antwerp Alessandro Murgia, University of Antwerp Quinten David Soetens, University of Antwerp

Serge Demeyer, University of Antwerp

\section{Steve Counsell, Brunel University \\ Xiaohui Liu, Brunel University \\ Stephen Swift, Brunel University \\ Jim Buckley, University of Limerick \\ Michael English, University of Limerick \\ Sebastian Herrold, University of Limerick \\ Sigrid Eldh, Ericsson \\ Andreas Ermedahl, Ericsson}

An Exploration of the 'Introduce Explaining Variable' Refactoring

The Evolution of Knowledge in the Refactoring Research Field

Matteo Orrù, University of Cagliari

Simone Porru, University of Cagliari

Michele Marchesi, University of Cagliari

Roberto Tonelli, University of Cagliari

Including structural factors into metrics-based code smells detection

Bartosz Walter, Poznan University of Technology

Błażej Matuszyk, Poznan University of Technology

Francesca Arcelli Fontana, University of Milano-Bicocca 


\title{
Program Committees
}

\section{3rd International Workshop on Large-Scale Agile Development}

\author{
Jan Bosch, Univeristy of Gothenburg, Sweden \\ Siva Dorairaj, Software Education, New Zealand \\ Tore Dybå, SINTEF ICT, Norway \\ Jutta Eckstein, IT Communication, Germany \\ Elke Hochmüller, Carinthia University of Applied Sciences, Austria \\ Maarit Laanti, Nitor Delta, Finland \\ Sridhar Nerur, University of Texas at Arlington, USA \\ Jaana Nyfjord, SICS Swedish ICT, Sweden \\ Orla O'Dwyer, Lero, Ireland \\ Helena Holmström Olsson, Malmö University, Sweden \\ Ken Power, Cisco, Ireland \\ Rini van Solingen, Delft University of Technology, the Netherlands \\ Roger Sweetman, Lero, Ireland \\ Hans van Vliet, Vrije Universiteit Amsterdam, the Netherlands
}

\section{1st International Workshop on Requirements Engineering in Agile Development (READ)}

\author{
Richard Berntsson Svensson, Chalmers | University of Gothenburg, Sweden \\ Elizabeth Bjarnason, Department of Computer Science, Lund University, Sweden \\ Lan Cao, Old Dominion University, USA \\ Tiago Silva Da Silva, ICT/UNIFESP - Universidade Federal de São Paulo - Instituto de Ciência e \\ Tecnologia, Brazil \\ Neil Ernst, Software Engineering Institute, USA \\ Smita Ghaisas, Tata Research Design and Development Center, India \\ Ville T. Heikkilä, Aalto University, Finland \\ Slinger Jansen, Utrecht University, Netherlands \\ Andrey Maglyas, Lappeenranta University of Technology, Finland \\ Tomi Männistö, University of Helsinki, Finland \\ Kari Smolander, Lappeenranta University of Technology, Finland \\ Xiaofeng Wang, Free University of Bozen-Bolzano, Italy
}




\section{International Workshop on Refactoring \& Testing (RefTest 2015)}

Giuseppe Destefanis, Computer Research Institute of Montreal, Canada

Alessandro Murgia, University of Antwerp, Belgium

Bartosz Walter, Poznań University of Technology, Poznań, Poland

Agustin Yague, Universidad Politécnica de Madrid, Spain

Marco Zanoni, University of Milano-Bicocca, Italy 